\author{
ID: DE-FG02-02ER63416 \\ Principal Investigator: Cesare Saltini \\ Institution: Department of Internal Medicine; University of Rome "Tor Vergata" \\ Title: Analysis of HLA-DP association with beryllium disease susceptibility in \\ pooled exposed populations \\ Report Type Final
}

\title{
Description of the objective of the project:
}

Berylliosis or Chronic Beryllium Disease is a chronic granulomatous disorder primarily involving the lung associated with the exposition to low doses of Beryllium $(\mathrm{Be})$ in the workplace. Berylliosis risk has been associated with the presence of a glutamate at position 69 of the HLA-DP beta chain (HLA-DPbetaGlu69) that is expressed in about $97 \%$ of disease cases and in $27 \%$ of the unaffected Be-exposed controls $(\mathrm{p}<0.0001)$ (Richeldi et al. Science 1993; 262: 242-244.12). Since this first observation of an immunogenetic association between berylliosis and HLA-DPbetaGlu69 a number of studies have confirmed the role of this marker as the primary gene of susceptibility of berylliosis (Richeldi et al Am J Ind Med. 1997; 32:337-40; Wang et al J. Immunol. 1999; 163: 1647-53; Saltini et al Eur Respir J. 2001 18:677-84; Rossman et al Am J Respir Crit Care Med. 2002 165:788-94). Moreover, a structure/function interaction between HLA-DP molecules carrying Glu69 and beryllium in driving and developing the immune response against beryllium itself has been observed as: (i) Be-specific T-cells clones obtained from berylliosis patients recognise beryllium as antigen only when presented in the context of the HLA-DP $\beta$ Glu69 molecules but not in the context of HLA-DP allelic variants carrying Lys69 (Lombardi G et al. J Immunol 2001; 166: 3549-3555), and (ii) beryllium presents an affinity for the HLA-DP2, carrying the berylliosis marker of susceptibility HLA-DPGlu69, from 40 to 100 times higher that the HLA-DP molecule carrying Lys69 (Amicosante M. et al Hum. Immunol. 2001; 62: 686-93).

However, although the immunogenetic studies performed have been addressed a number of different questions about the genetic association between berylliosis and/or beryllium sensitization, exposure levels to beryllium and HLA markers, a number of questions are still open in the field mainly due to the limitation imposed by the low number of subjects carrying berylliosis or beryllium sensitization enrolled in each immunogenetic study.

\section{Description of the approach employed in the project}

In this context, the populations of the study already performed in this field by the University of Modena and Rome (by Prof. C. Saltini) and the University of Pennsylvania (by Prof. M. Rossman) have been evaluated by using similar HLA molecular typing methodologies and that both populations have now been followed up for a period of 4 to 7 years.

The general objective of this study has to generate a larger data base comprising the two population with which analyze gene disease association with greater statistical power and ascertain the effect of lesser common gener variants which may be missed when analyzing associations on small populations. In particular addressing the role suggested in previous study such as: (i) the role of HLA-DP rare alleles and polymorphisms, and (ii) the role of the HLA markers in disease progression from sensitization.

\section{Final report results:}


The two populations from the already published studies (Saltini et al Eur Respir J. 2001 18:677-84; Rossman et al Am J Respir Crit Care Med. 2002 165:788-94) present similar aspects about: ethnicity, type and length of exposure to Be dust, a broadly similar association between beryllium related abnormalities and HLA. The two population have been pooled and evaluated using common criteria of diagnosis (Sensitized subject: at least 2 positive BeLPT tests each with 2 positive wells; CBD-affected subject: identification of well formed non-caseating granulomas on biopsy), follow up and HLA typing technique (complete HLA-DRB, DQB, DPB high resolution typing using amplification with sequence specific primers or sequence based typing).

The two populations included 137 subjects with Beryllium hypersensitized (BH) and $155 \mathrm{Be}-$ exposed controls. Inclusion criteria were met by one hundred and six subjects with Behypersensitivity of whom 55 were affected by CBD (age $52 \pm 11$ years; 50 caucasians, 2 AfricanAmericans 2 Hispanics and 1 Asian; 46 males and 9 females; mean duration of Be-exposure 15+9 years) and 51 showed Be-sensitization without lung granulomas detected by trans-bronchial biopsy (age 54+14 years; 47 Caucasians, 3 Hispanics and 1 Afro-American; 45 males and 6 females; mean age of Be-exposure $17 \pm 10$ years) and 129 Be-exposed controls (age $53 \pm 14$ years; 120 Caucasians, 4 African-American, 4 Hispanics, 1 Asian; 104 males and 25 females; mean duration of Be-exposure $16 \pm 10$ years). Mean follow-up of $\mathrm{BH}$ affected subjects was $7.0 \pm 3.7$ years from the first positive BeLPT test.

\section{HLA-DPB1, -DQB1, -DRB1, -DRB3, -DRB4 and -DRB5 phenotypic frequencies in $B H$}

We first analyzed the association of HLA-DPB1, -DQB1, -DRB1, -DRB3, -DRB4 and -DRB5 polymorphic aminoacid residues with $\mathrm{BH}$ by performing univariate analysis on the phenotypic frequency of all aminoacid variants present in the 108 polymorphic residues of the HLA class II $\beta$ chains. Table I shows all the aminoacid variants which were found positively or negatively associated with $\mathrm{BH}$ in comparison to Be-exposed controls. The already defined $\mathrm{BH}$ susceptibility marker DPGlu69 was present in 54 out of 129 (42\%) Be-exposed controls and in 85 out of 106 $(80 \%, \mathrm{OR}=5.6, \mathrm{p}<0.000001)$ subjects with $\mathrm{BH}$. No differences were observed between $\mathrm{BH}$ subjects with and without lung granulomas in the distribution of DPGlu69 [CBD 47/55 (85\%), BH without granuloma 38/51 (75\%), $\mathrm{p}>0.05$ ]. In addition, the frequency of DPGlu69 homozygous subjects was similar in the two groups (data not shown). Four other HLA-DPB1 aminoacid variants (Leu11, Val36, Asp55 and Ile76) were found associated with BH (Table I). Since all of these residues could be in linkage disequilibrium, forward and stepwise multiple logistic regression models were carried out to evaluate the association of each aminoacid variant with the study groups. The logistic models showed that, of all the polymorphisms associated with Be-hypersensitivity, only DPGlu69 was independently associated with $\mathrm{BH}$ (OR 2.895, $\mathrm{p}<0.05)$. Furthermore, the single linkage disequilibrium analysis between HLA-DPGlu69 and the other HLA-DP aminoacid residue markers confirmed this result. In fact, HLA-DPGlu69 (overall frequency 0.351) resulted in linkage disequilibrium with all the other residues found associated: Leu11 (overall frequency 0.188, expected frequency of independent recombination with HLA-DPGlu69 0.066, observed frequency of HLA-DPGlu69 and Leu11 double positives 0.104, p=0.0469), Val36 (overall frequency 0.557, expected frequency of independent recombination with HLA-DPGlu69 0.195, observed frequency of HLA-DPGlu69 and Val36 double positives 0.317, p<0.00001), Asp55 (overall frequency 0.487, expected frequency of independent recombination with HLA-DPGlu69 0.171, observed frequency of HLA-DPGlu69 and Asp55 double positives 0.300, p<0.00001) and Ile76 (overall frequency 0.040, expected frequency of independent recombination with HLA-DPGlu69 0.014, observed frequency of HLA-DPGlu69 and Ile76 double positives 0.040, $\mathrm{p}=0.0150$ ).

With regard to HLA-DQB1, nine aminoacid variants (Leu26, Pro55 and the clustered residues Gln84, Leu85, Glu86, Leu87, Thr89 and Thr90) were either positively or negatively associated with BH in the univariate analysis (Table I). The logistic models showed that of all the HLA-DQ 
polymorphisms associated with BH only HLA-DQ-Leu26 was independently associated (OR 2.312, $\mathrm{p}<0.05$ ) with $\mathrm{BH}$.

Similarly, seven HLA-DRB1 aminoacid variants (Val11, His32, His33, Asn37, Tyr47, Phe47 and Asn77) were positively or negatively associated with BH in the univariate analysis (Table I). Once again, we applied forward and stepwise multiple logistic regression models to evaluate the association of aminoacid variants with study groups as all these residues could be in linkage disequilibrium. The logistic models showed that only the polymorphic variants HLA-DRPhe47 and HLA-DRAsn77, among the HLA-DRB1 locus variants, were independently associated with BH (Phe47: OR 2.289, $\mathrm{p}<0.05$; Asn77: OR 1.872, $\mathrm{p}<0.05$ ).

No polymorphisms associated to BH were found in the HLA-DRB3, -DRB4 and DRB5 loci in the univariate analysis.

Finally, when all the aminoacid variants found associated with $\mathrm{BH}$ were evaluated in multiple logistic regression models only HLA-DPGlu69, HLA-DRPhe47 and HLA-DRAsn77 were independently associated with hypersensitivity to Be.

\section{HLA-DP markers and disease progression.}

The HLA-DPGlu69 has been previously proposed as a marker of progression from the sensitization state to the lung granulomatous reaction of chronic beryllium disease $(10,13)$. In this study population there were no cases of progression from systemic sensitization to lung disease over a follow-up period of 7.0+3.7 years from the first positive Be-LPT test. We therefore looked at the frequency of HLA-DPGlu69 homozygous subjects among the BH affected, with or without disease. There was an increase in HLA-DPGlu69 homozygosity in the population of BH subjects [12 out of 129 healthy exposed controls $(9.3 \%), 5$ out of 51 sensitized without disease $(9.8 \%$; $\mathrm{OR}=1.1, \mathrm{p}=0.858$ compared to controls $)$ and 9 out of 55 disease affected $(16.4 \% ; \mathrm{OR}=1.9, \mathrm{p}=0.131$ compared to controls; $\mathrm{OR}=1.8 \mathrm{p}=0.475$ compared to the sensitized)] that however was not statistically significant.

HLA-DPB1, -DQB1, -DRB1, -DRB3, -DRB4 and -DRB5 phenotypic frequencies in HLADPGlu69-negatives BH subjects.

Twenty-one $\mathrm{BH}$ and $75 \mathrm{Be}$-exposed controls resulted DPGlu69-negative; they did not differ from the DPGlu69-positive, either the BH affected or the Be-exposed controls, subjects in terms of gender, ethnicity, age or length of Be-exposure ( $p>0.05$, all comparisons). This subgroup of HLADPGlu69 negative subjects was analyzed for the distribution of all HLA class II polymorphic aminoacid residues, with the exception of HLA-DPGlu69, by univariate analysis. No associations were found between any of the HLA-DP polymorphic residues and BH. Strikingly, among the polymorphic residues of the HLA-DR $\beta$-chain coded for by the HLA-DRB1 locus, residues His32, Asn37 and Phe47 were associated with BH (Table II). No polymorphisms associated with BH were found in the HLA-DRB3, -DRB4 and DRB5 loci.

Since the HLA-DRB1 gene polymorphisms His32 and Asn37 are also expressed by HLA-DRB3 and HLA-DRB5 alleles, we evaluated their frequency in the context of all of the HLA-DRB1, DRB3, DRB5 loci polymorphisms. This extended analysis showed no association for residue His32 $(16 / 21,76 \%$; Be-exposed controls 46/75, 61\%, $\mathrm{p}=0.32, \mathrm{OR}=2.0)$ while the association of residue Asn 37 with BH remained statistically significant (15/21, 71\%; Be-exposed controls 33/75, 44\%, $\mathrm{p}=0.048, \mathrm{OR}=3.2$ ). However, a linkage disequilibrium could be demonstrated between residues Asn 37 and Phe47. With the overall allelic frequencies of $27.6 \%$ for Asn 37 and of $52.5 \%$ for Phe47, the expected frequency of independent recombination for Asn37 and Phe47 was 14.5\%. Instead, the observed frequency of $25.3 \%$ Asn37/Phe47 double positives $(p<0.0001)$ suggested a carryover effect.

Finally, although a statistically significant association with BH was found for the HLA-DQB1 gene polymorphic residue Leu26 (Table II), when multiple logistic regression models were carried out on the three variants (HLA-DRAsn37, HLA-DRPhe47 and HLA-DQLeu26), only HLA-DRPhe47 (OR 
2.879, $\mathrm{p}<0.05$ ) resulted independently associated with BH in the HLA-DPGlu69-negative subjects, thus suggesting the presence of linkage disequilibrium between HLA-DRPhe47 and both HLADQLeu26 and HLA-DRAsn37.

HLA class II restriction of Be-stimulated T-cell proliferation of HLA-DPGlu69-negative BH affected subjects

In order to identify the HLA isotypic molecule(s) functioning as the restriction elements of Bestimulated T-cell proliferation in the HLA-DPGlu69-negative subjects, the ability of antibodies directed against HLA-DR, HLA-DQ and HLA-DP to inhibit PBMC proliferation in response to $\mathrm{BeSO}_{4}$ was assessed in a subgroup of $19 \mathrm{BH}$ affected subjects, among which 15 showed T-cell proliferation with a twofold or greater stimulation index.

In the four HLA-DPGlu69-negatives, Be-stimulated T-cell proliferation was inhibited by the antiHLA-DR MoAb (range 70-92\% inhibition) significantly more than by the anti-HLA-DP MoAb (range: $6-29 \% ; \mathrm{p}<0.02$ compared to anti-HLA-DR) while it was not affected at all by the anti-HLADQ, anti-HLA class I or the anti-MTB19 control antibody (Figure 1), suggesting a role for the HLA-DR molecule in the presentation of Be in the HLA-DPGlu69-negative subjects. All the four HLA-DPGlu69-negative subjects carried the HLA-DRPhe47 polymorphism. In addition, three out of four also carried the HLA-DRAsn37 residue.

In contrast, in the three subjects who were HLA-DPGlu69 positive and HLA-DRPhe47/HLADRAsn37 negative, proliferation was completely inhibited by the anti-HLA-DP MoAb (range 68$100 \%$ ) but not by anti-HLA-DR (range $7-14 \%, \mathrm{p}<0.05$ compared to anti-HLA-DP), nor by antiHLA-DQ, anti-HLA class I or the anti-MTB19 control antibody (Figure 1). Finally, in the eight subjects carrying both HLA-DPGlu69 and HLA-DRPhe47 (with or without HLA-DRAsn37), the proliferative response to $\mathrm{BeSO}_{4}$ was always inhibited by the anti-HLA-DP (range: 63-100\%) and variable inhibited by anti-HLA-DR antibodies (range: 0-94\%), with the inhibition by anti HLA-DP being significantly stronger than the anti HLA-DR (paired t-test, $\mathrm{p}<0.01$ ). 
Table I. Phenotypic frequencies of the HLA class II polymorphisms found associated with Behypersensitivity

\begin{tabular}{|c|c|c|c|c|}
\hline & $\begin{array}{l}\text { Be-exposed controls } \\
(\mathrm{n}=129)\end{array}$ & $\begin{array}{l}\text { Be-Hypersensitive } \\
(\mathrm{n}=106)\end{array}$ & & \\
\hline \begin{tabular}{|l|} 
HLA-DP \\
polymorphism
\end{tabular} & $\begin{array}{l}N \text { positive subjects } \\
(\%)^{2}\end{array}$ & $\begin{array}{l}\text { N positive subjects } \\
(\%)^{2}\end{array}$ & $\mathbf{O R}^{3}$ & $p^{4}$ \\
\hline Leu11 & $39(30.23 \%)$ & $48(45.28 \%)$ & 1.9 & 0.025 \\
\hline Val36 & $99(76.74 \%)$ & $99(93.40 \%)$ & 4.3 & 0.0009 \\
\hline Asp55 & $90(69.77 \%)$ & $87(82.08 \%)$ & 2.0 & 0.0294 \\
\hline \begin{tabular}{|l|} 
Glu69 \\
\end{tabular} & $54(41.86 \%)$ & $85(80.19 \%)$ & 5.6 & $<0.00001$ \\
\hline Ile76 & $5(3.88 \%)$ & $14(13.21 \%)$ & 3.8 & 0.0178 \\
\hline $\begin{array}{l}\text { HLA-DQ } \\
\text { polymorphism }^{5}\end{array}$ & & & & \\
\hline Leu26 & $80(64.10 \%)$ & $82(78.10 \%)$ & 2.1 & 0.0117 \\
\hline Pro55 & $79(61.24 \%)$ & $52(49.06 \%)$ & 0.6 & 0.0425 \\
\hline$G \ln 84$ & $108(86.40 \%)$ & $79(75.24 \%)$ & 0.6 & 0.0439 \\
\hline Leu85 & $108(86.40 \%)$ & $79(75.24 \%)$ & 0.6 & 0.0439 \\
\hline Glu86 & $108(86.40 \%)$ & $79(75.24 \%)$ & 0.6 & 0.0439 \\
\hline Leu87 & $108(86.40 \%)$ & $79(75.24 \%)$ & 0.6 & 0.0439 \\
\hline Thr89 & $108(86.40 \%)$ & $79(75.24 \%)$ & 0.6 & 0.0439 \\
\hline Thr90 & $108(86.40 \%)$ & $79(75.24 \%)$ & 0.6 & 0.0439 \\
\hline $\begin{array}{l}\text { HLA-DR } \\
\text { polymorphism }^{6}\end{array}$ & & & & \\
\hline Val11 & $31(24.03 \%)$ & $8(7.55 \%)$ & 0.3 & 0.0017 \\
\hline His32 & $57(44.19 \%)$ & $62(58.49 \%)$ & 1.8 & 0.0402 \\
\hline His33 & $43(33.33 \%)$ & $15(14.15 \%)$ & 0.3 & 0.0102 \\
\hline Asn37 & $48(37.21 \%)$ & $58(54.72 \%)$ & 2.0 & 0.0107 \\
\hline Tyr47 & $75(58.14 \%)$ & $43(40.57 \%)$ & 0.5 & 0.0108 \\
\hline Phe47 & 87 (67.44\%) & $85(80.19 \%)$ & 2.0 & 0.0406 \\
\hline Asn77 & $21(20.93 \%)$ & $30(28.30 \%)$ & 2.0 & 0.0388 \\
\hline
\end{tabular}


Notes:

1. HLA-DPB1 polymorphisms found associated with Be-hypersensitivity among the overall HLA-DPB1 polymorphic variant analyzed at positions: $8,9,11,33,35,36,55,56,57,65$, $69,72,76,84,85,86$ and 87.

2. Number of subjects carrying the reported polymorphic variant in the study population and phenotypic frequency in bracket.

3. Odds ratio with respect to Be-exposed controls.

4. $\quad \mathrm{p}$ value $\left(\chi^{2}\right.$ analysis with Yates' correction) with respect to Be-exposed controls.

5. HLA-DQB1 polymorphisms found associated with Be-hypersensitivity among the overall HLA-DPQ1 polymorphic variant analyzed at positions 9, 13, 14, 23, 26, 28, 30, 37, 38, $45,46,47,49,52,53,55,56,57,60,66,67,70,71,74,75,77,84,85,86,87,89$ and 90.

6. HLA-DRB1 polymorphisms found associated with Be-hypersensitivity among the overall HLA-DRB1 polymorphic variant analyzed at positions $9,10,11,12,13,16,26,28,30$, $31,32,33,37,38,40,47,57,58,59,60,67,70,71,72,73,74,77,85$ and 86 
Table II. Phenotypic frequencies of the polymorphisms found associated with Be-hypersensitivity in HLA-DPGlu69 negative subjects.

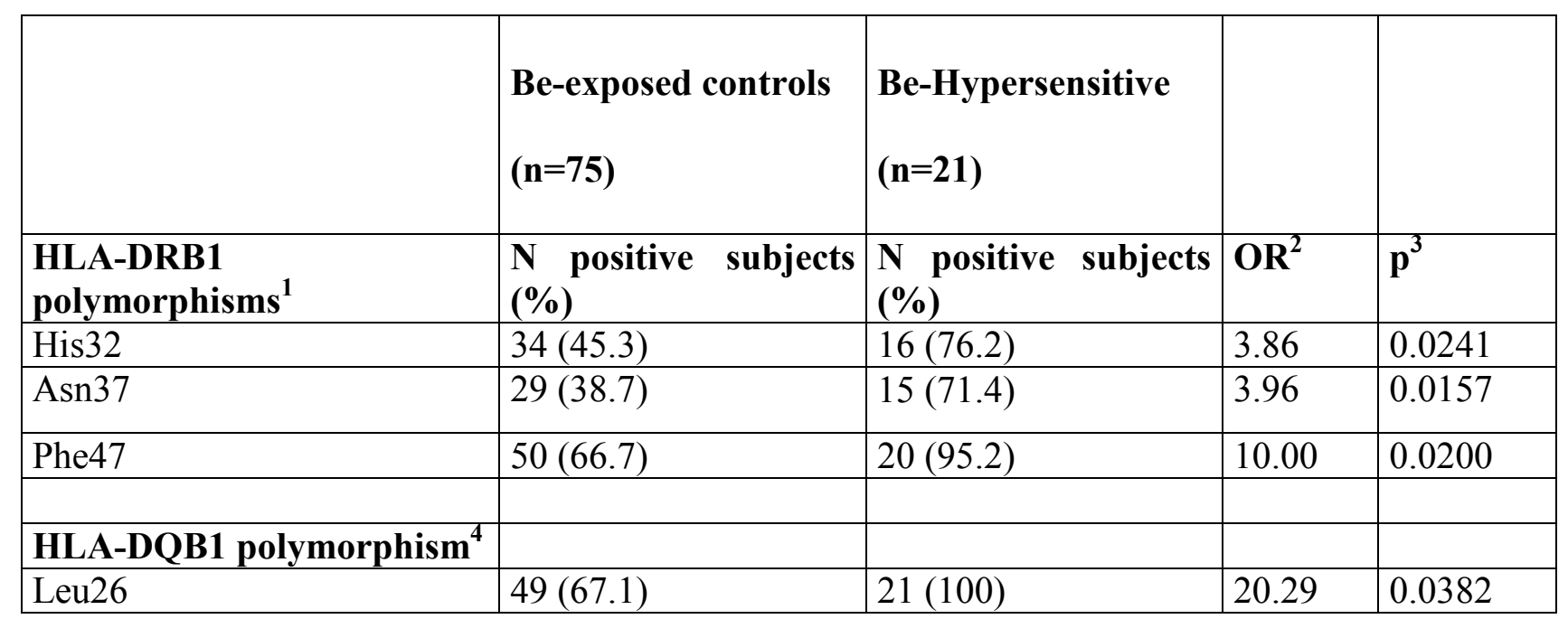

Notes:

1. HLA-DRB1 polymorphisms found associated with Be-hypersensitivity in HLA-DPGlu69 negative subjects among the overall HLA-DRB1 polymorphic variants analyzed at positions: $9,10,11,12,13,16,26,28,30,32,37,38,47,57,58,60,67,70,71,73,74,77$, 85,86 .

2. Odds ratio with respect to Be-exposed controls.

3. $\mathrm{p}$ value ( $\chi^{2}$ analysis) with respect to Be-exposed controls.

4. HLA-DQB1 polymorphisms found associated with Be-hypersensitivity in HLA-DPGlu69 negative subjects among the overall HLA-DQB1 polymorphic variants analyzed at positions: $9,13,14,23,26,28,30,37,38,45,46,47,52,53,55,56,57,66,67,70,71,74$, $75,77,84,85,86,87,89,90$. No polymorphisms were found associated to BH in the HLADRB3, -DRB4 and DRB5 loci and HLA-DP locus. 


\section{FIGURE LEGENDS}

Figure 1. Inhibition of beryllium $\left(\mathrm{BeSO}_{4}\right)$-induced proliferation, by MoAbs directed against HLADR, HLA-DP, HLA-DQ, HLA-class I and the $19 \mathrm{kDa}$ M. tuberculosis protein in PBMC from BH subjects carrying or not the HLA-DPGlu69 and the HLA-DRPhe47 markers. On the ordinate is shown the percentage of inhibition (with respect to the MoAbs untreated cells) of T-cell proliferation obtained by co-culturing the PBMC from berylliosis patients with $\mathrm{BeSO}_{4}$ in the presence of each MoAb reported on the abscissa (anti-HLA-DR: DR, anti-HLA-DP: DP, anti-HLADQ: DQ, anti-HLA-class I: C.I, anti-19 kDa M. tuberculosis: Mtb).

Figure 2. Analysis of the electron donor groups present in the pocket 4 and 9 of HLA-DP molecules. Panel A, shows the molecular model of HLA-DP0201 (28) presenting together with Glu69 other electron donor groups such as Gln $\beta 13, \operatorname{Glu} \beta 14, \operatorname{Arg} \beta 27$ and $\operatorname{Tyr} \beta 28$. Specifically, at least four oxygens belonging to the Gln13, Glu14 and Glu69 are free of coordinating Be. Similarly in pocket 9, HLA-DP0201 molecule presents Asp $\beta 55$ and Arg $\alpha 74$ that could play a role in coordinating Be, while Glu $\beta 56$ is oriented outside the binding groove. Panel B shows the molecular model of HLA-DP0402 (28) presenting Lys in position 69 of the $\beta$-chain instead of Glu as the only difference with HLA-DP0201. In this molecule, although a number of electron donor groups are present, the capability to coordinate Be ion in pocket 4 is reduced respect to HLA-DP0201 by the presence of positive charged residues that engages the negatives groups in a different H-bond network. The capability of binding Be in pocket 9 of HLA-DP0402 is similar to that of HLADP0201. Panel C report the molecular model of HLA-DP0401. Pocket 4 present the same features of the HLA-DP0402 pocket 4. Moreover, in pocket 9 the change of Asp with Ala in position $\beta 55$ determine a further reduced capability to coordinate Be. Molecular models have been evaluated with the SwissPDB viewer. The HLA-DP $\alpha$-chain backbone is reported in red colored ribbon style, while the HLA-DP $\square$-chain backbone is reported in grey colored ribbon. Aminoacids are colored in CPK style (C: light blue; O: red; $\mathrm{N}$ : blue) and residue names are reported in red. $\mathrm{H}$-bonds were computed with the SwissPDB viewer and are shown as green dashed lines.

Figure 3. Analysis of the H-bond network in the pocket 7 of HLA-DR molecules carrying HLADRPhe47 (Panel A: HLA-DR3 and Panel B: HLA-DR15) or its counterpart Tyr47 (Panel C: HLADR1 and Panel D: HLA-DR4). Electron donor groups present in pocket 7 of HLA-DR1 (PDB entry: 1AQD), HLA-DR3 (1A6A), HLA-DR4 (2SEB) and HLA-DR15 (1BX2), putatively capable of coordinating $\mathrm{Be}$ are reported (residues $\alpha 69, \beta 28, \beta 61, \beta 70$ and $\beta 71$ ). In the HLA-DR3 crystal structure (Panel A) with the presence of Phe 47 only one of the two terminal oxygens of Asp $\beta 28$ is engaged in a H-bond network with Lys71, leaving four other contacts points for co-ordinating Be. A similar pattern is present in HLA-DR15 (Panel B) where, with the presence of Phe47, no H-bonds are present leaving 5 electron donor groups available for Be coordination. When Tyr47, the HLADRPhe47 counterpart, is present in HLA-DR molecules as in HLA-DR1 (panel C) and HLA-DR4 (Panel D), the H-bond network of pocket 7 results dramatically modified. Specifically, Tyr47 engages in a H-bond network with residues Asp28 and Arg71 in HLA-DR1 (Panel C) or Asp28 and Lys71 in HLA-DR4 (Panel D). As a consequence there is reduced availability of electron donor groups able to coordinate Be. Molecular modelling of the PDB entry crystal structures have been evaluated with the SwissPDB viewer. The HLA-DR $\alpha$-chain backbone is reported in red colored ribbon style, while the HLA-DR $\beta$-chain backbone is reported in grey colored ribbon. Aminoacids are colored in $\mathrm{CPK}$ style $(\mathrm{C}$ : light blue; $\mathrm{O}$ : red; $\mathrm{N}$ : blue) and residue names are reported in red. $\mathrm{H}-$ bonds were computed with the SwissPDB viewer and are shown as green dashed lines. 
Figure 1

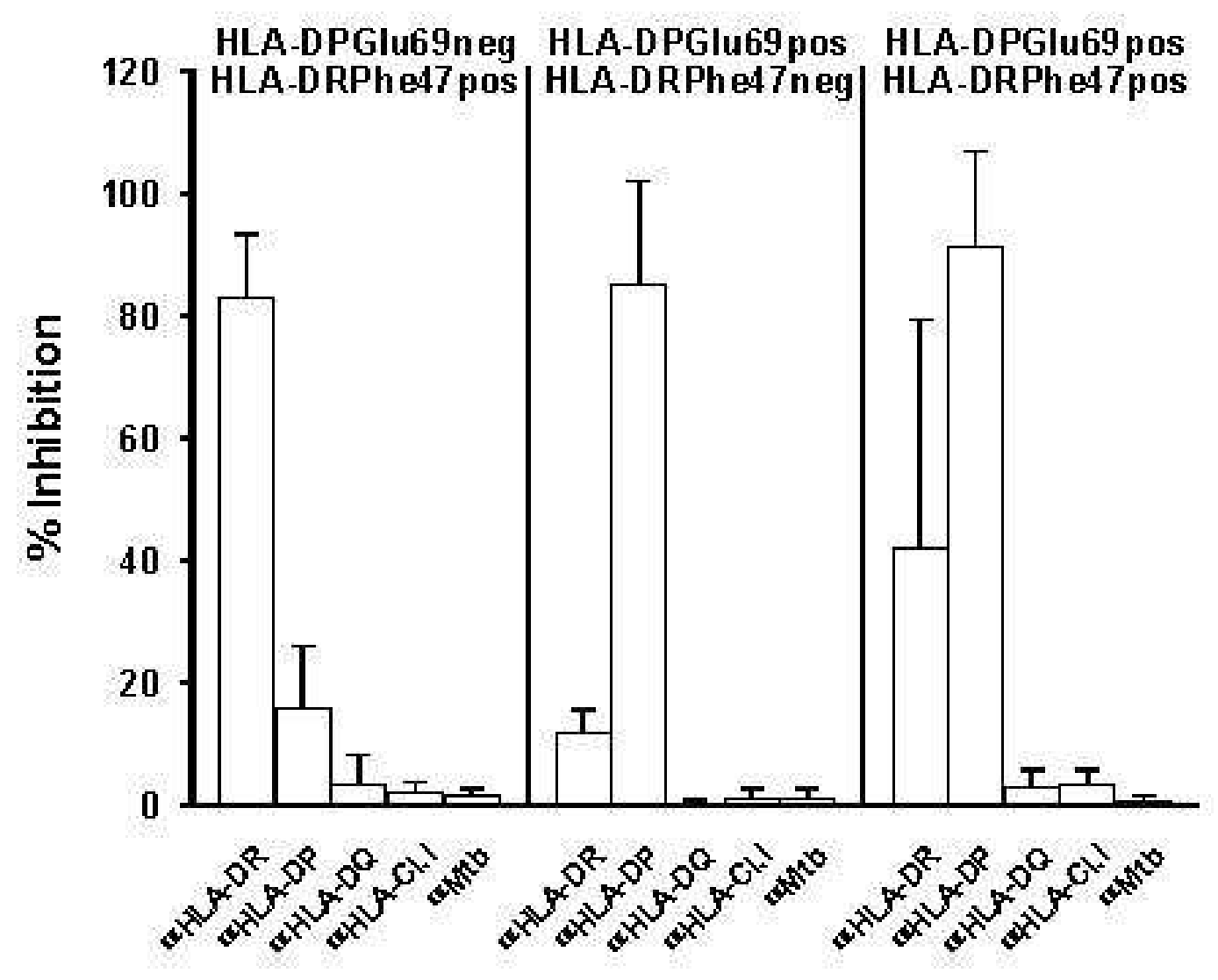


Figure 3

A. HLA-DR3

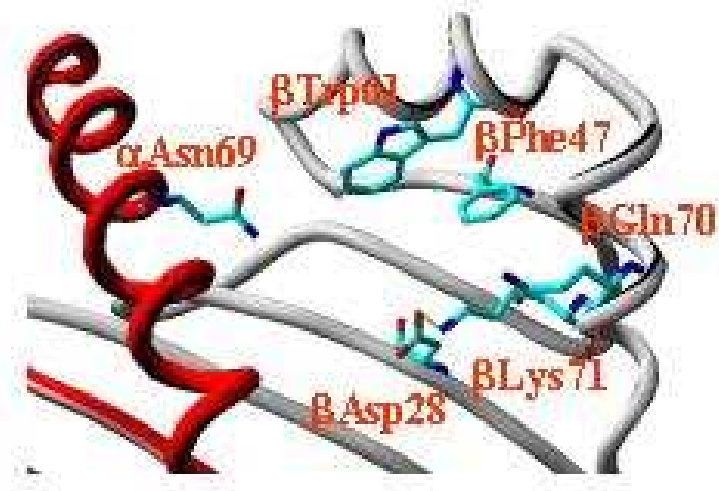

C. HLA-DR1

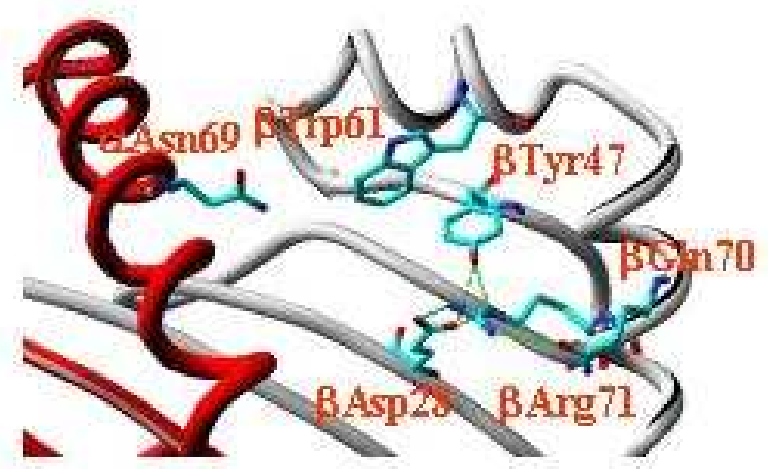

B. HLA-DR15



D. HLA-DR4

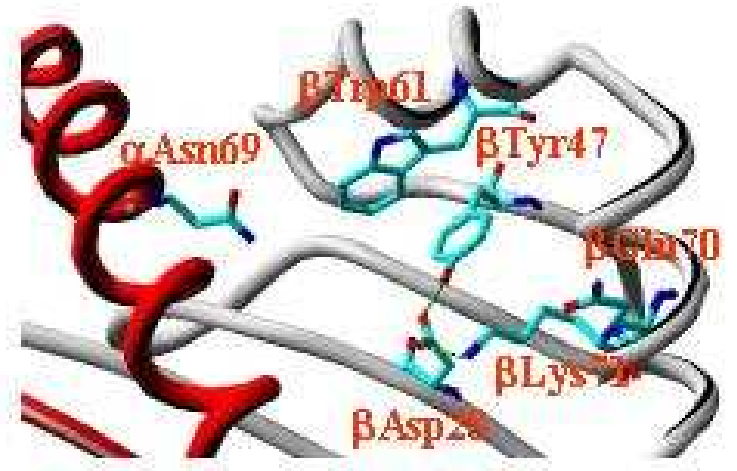


Figure 2

A. HLA-DPB1 *0201

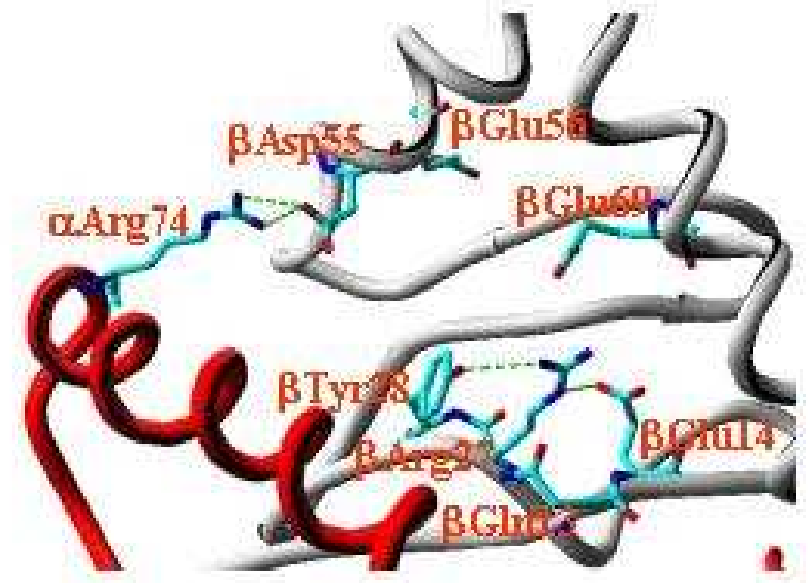

\section{B. HLA-DPB1 * 0402}

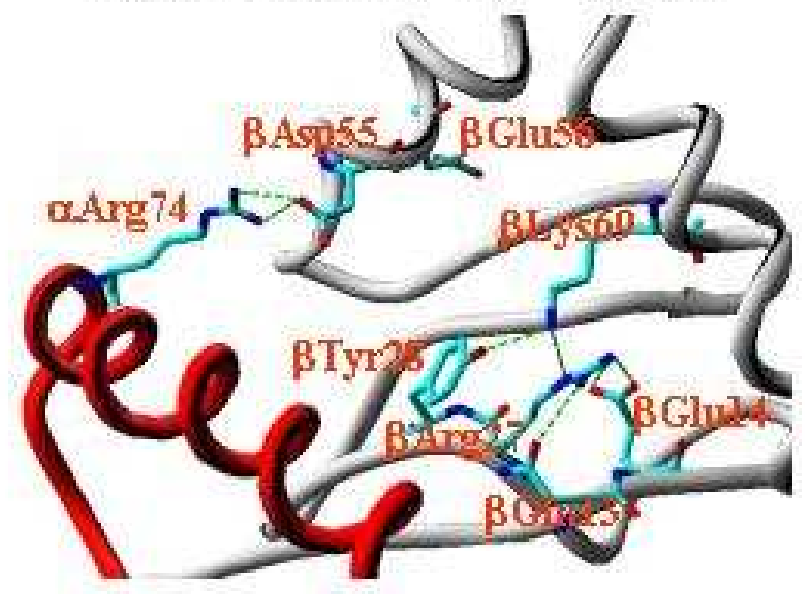

C. HLA-DPB1 *0401

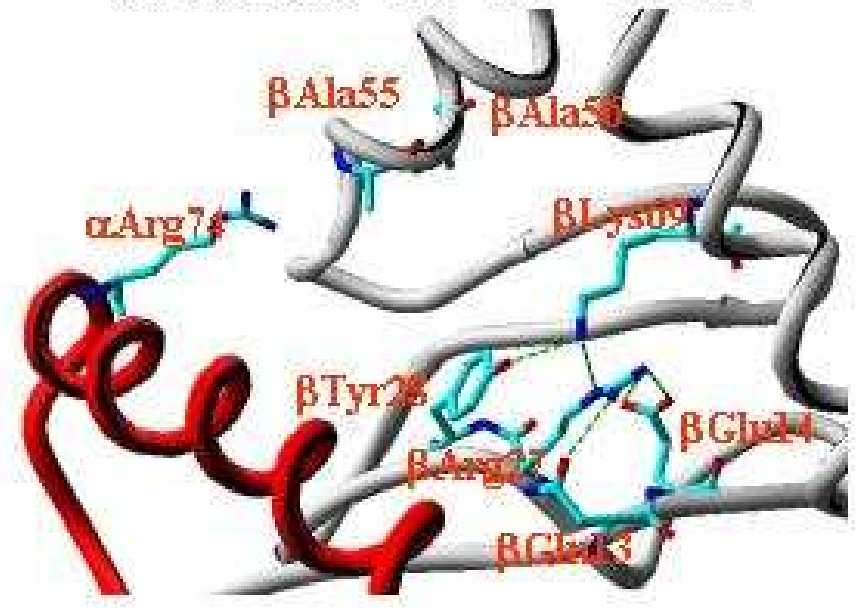




\section{Products delivered:}

Gaede K.I., Amicosante M., Schurmann M., Fireman E., Saltini C., Muller-Quernheim J. Function associated transforming growth factor-beta gene polymorphism in chronic beryllium disease. J. Mol. Med. 2005; 83: 397-405.

Amicosante M., Berretta F., Rossman M., Butler R.H., Rogliani P., van den Berg-Loonen E., Saltini C. Identification of HLA-DRPhebeta47 as the susceptibility marker of hypersensitivity to beryllium in individuals lacking the berylliosis-associated supratypic marker HLA-DPGlubeta69. Respir Res. 2005; 6: 94.

Amicosante M., Deubner D., Saltini C. Role of the berylliosis-associated HLA-DPGlu69 supratypic variant in determining the response to beryllium in a blood T-cells beryllium-stimulated proliferation test. Sarcoidosis Vasc. Diffuse Lung Dis. 2005; 22: 175-9.

Amicosante M, Berretta F, Dweik R, Saltini C. Role of high-affinity HLA-DP specific CLIPderived peptides in beryllium binding to the HLA-DPGlu69 berylliosis-associated molecules and presentation to beryllium-sensitized $\mathrm{T}$ cells. Immunology. 2008; 128: e462 - e470. DOI: 10.1111/j.1365-2567.2008.03000.x. 